\title{
Screening tools for common mental disorders in older adults in South Asia: a systematic scoping review
}

\author{
Lachlan Fotheringham, ${ }^{1}$ (i) Stella-Maria Paddick, ${ }^{2}$ Evelyn Barron Millar, ${ }^{3}$ \\ Claire Norman, ${ }^{1}$ Ammu Lukose, ${ }^{4}$ Richard Walker, ${ }^{5}$ and Mathew Varghese ${ }^{6}$ \\ ${ }^{1}$ Cumbria, Northumberland, Tyne and Wear NHS Foundation Trust, Newcastle Upon Tyne, UK \\ ${ }^{2}$ Newcastle University, Translational and Clinical Medicine, Newcastle Upon Tyne, UK \\ ${ }^{3}$ Wolfson Research Centre, Campus for Ageing and Vitality, Newcastle University, Newcastle upon Tyne, UK \\ ${ }^{4}$ Centre For Community Mental Health (CCMH), Mangalore, India \\ ${ }^{5}$ Northumbria Healthcare NHS Foundation Trust, Department of Medicine, North Shields, UK \\ ${ }^{6}$ National Institute of Mental Health $\mathcal{G}$ Neuro Sciences (NIMHANS), Bangalore, India
}

ABSTRACT

Objectives: Common mental disorders (CMDs), particularly depression, are major contributors to the global mental health burden. South Asia, while diverse, has cultural, social, and economic challenges, which are common across the region, not least an aging population. This creates an imperative to better understand how CMD affects older people in this context, which relies on valid and culturally appropriate screening and research tools. This review aims to scope the availability of CMD screening tools for older people in South Asia. As a secondary aim, this review will summarize the use of these tools in epidemiology, and the extent to which they have been validated or adapted for this population.

Design: A scoping review was performed, following PRISMA guidelines. The search strategy was developed iteratively in Medline and translated to Embase, PsychInfo, Scopus, and Web of Science. Data were extracted from papers in which a tool was used to identify CMD in a South Asian older population $(50+)$, including validation, adaptation, and use in epidemiology. Validation studies meeting the criteria were critically appraised using the Quality Assessment of Diagnostic Accuracy Studies - version 2 (QUADAS-2) tool.

Results: Of the 4694 papers identified, 176 met the selection criteria at full-text screening as relevant examples of diagnostic or screening tool use. There were 15 tool validation studies, which were critically appraised. Of these, 10 were appropriate to evaluate as diagnostic tests. All of these tools assessed for depression. Geriatric Depression Scale (GDS)-based tools were predominant with variable diagnostic accuracy across different settings. Methodological issues were substantial based on the QUADAS-2 criteria. In the epidemiological studies identified $(n=160)$, depression alone was assessed for $82 \%$ of the studies. Tools lacking cultural validation were commonly used (43\%).

Conclusions: This review identifies a number of current research gaps including a need for culturally relevant validation studies, and attention to other CMDs such as anxiety.

Key words: psychogeriatrics, depression, anxiety, epidemiology, screening

\section{Introduction}

The world population is aging rapidly, particularly in low- and middle-income countries (LMICs) compared with high-income countries (HICs) (UN 2015), and while South Asia has in the past lagged behind other parts of the world in this, its

Correspondence should be addressed to: Stella-Maria Paddick, Campus for Ageing and Vitality, Newcastle University, Westgate Road, Newcastle upon Tyne NE4 6BE, UK. Email: stella-maria.paddick@ncl.ac.uk. Received 05 Jun 2020; revision requested 22 Jun 2020; revised version received 06 Oct 2020; accepted 09 Oct 2020. First published online 08 January 2021. fertility rate is declining and life expectancy is climbing, bringing on a rapid demographic transition (Chand, 2018). In India, which accounts for most of the region's population, $8.6 \%$ of the population are aged 60 and over, a proportion projected to reach $19 \%$ by 2050 (Agarwal et al., 2016). The Lancet Global Mental Health series (2007 and 2011) highlighted the wide gap between mental health needs and provision (Eaton et al., 2011), but this gap is not well characterized in older people. Common mental disorders (CMDs), which 
encompass depression, anxiety, and related disorders, are a large part of the global disease burden, contributing substantially to morbidity, mortality, and decreased quality of life for people of all ages (Hay et al., 2017), but particularly in older people (Guerra et al., 2016; Ogbo et al., 2018).

There is a need to focus on older people across LMICs, but also on South Asia in particular. The majority of existing data in this area focus on HICs, with a relative lack in older people and LMICs. Initiatives such as the 10/66 International Dementia Research Collaboration (Prince et al., 2007) and the Study on Global Ageing and Adult Health (SAGE) (Kowal et al., 2012) have greatly added to the understanding of mental disorders in older people in LMICs, however, they still leave much to learn. The work of the 10/66 Collaboration is primarily focused on dementia, and SAGE aims to provide a broad representation from different geographic regions across LMICs (Lotfaliany et al., 2019), rather than a sharply focused impression of any one region in particular. While it can be useful to examine an issue in relation to income status, for example, LMICs, this risks conflating a range of very distinct cultural contexts. South Asian countries are often considered together in large meta-analyses, citing challenges common across the region, such as lack of access to resources, stigma, and underdeveloped mental health legislation, policies, and plans (Hossain et al., 2020; Ogbo et al., 2018; Ranjan and Asthana, 2017). There may also be common psychosocial constructs across the region (Rama et al., 2014), suggesting that it is useful to consider South Asia as a whole in a review such as this.

Appropriate screening tools play an important role in the identification and characterization of mental health needs. They aid assessment in routine clinical practice, facilitate epidemiological work, which in turn, informs policymakers in allocating scarce resources appropriately according to need. There are well-known effects of culture on presentations of mental disorders and idioms of distress (Kerr, 2001), and evidence that mental disorders can present differently in older people (Yesavage et al., 1982). It is currently not known, however, to what extent appropriate screening tools or measures for CMDs have been validated in South Asia, and where exactly the gaps in availability and validation of these measures are.

This scoping review aims to identify the extent to which screening tools for CMDs have been validated in older adults in South Asia, and to identify key research gaps and priorities for future work through performing a systematic search of the literature including validation studies, epidemiological studies, and existing literature reviews. The quality of the current validation data were assessed through the quality assessment of included validation studies using the Quality Assessment of Diagnostic Accuracy Studies - version 2 (QUADAS-2) (Whiting et al., 2011).

As a secondary aim, this scoping review will summarize the screening tools currently, and previously, being used in epidemiological studies of CMDs in older adults in South Asia and whether these have been previously validated and locally developed or culturally adapted.

The need for this review was identified by leading older people's mental health clinicians and researchers from South Asia at the inaugural meeting of North East England South Asia Mental health Alliance (NEESAMA) in November 2018. Mental health of older adults is a key theme of this initiative.

\section{Methods}

\section{Search strategy}

A systematic literature search was performed in July 2019 in the following databases:

Medline, Embase, PsycInfo, Scopus, and Web of Science. The search strategy was developed iteratively in Medline and translated across the remaining databases. To capture CMDs, we combined the keywords, "depression" OR "affective" OR "anxiety" OR "GAD" OR "panic" OR "phobia*” OR "obsessive" OR "compulsive" OR "OCD” OR "trauma" OR "PTSD" with the Medline subject headings, "ANXIETY DISORDERS"/ OR "MOOD DISORDERS"/ OR "NEUROTIC DISORDERS"/ OR "TRAUMA AND STRESSOR RELATED DISORDERS"/ OR "MENTAL HEALTH"/. These were combined with thesaurus and free-text terms to capture older adults, and countries in the South Asia region.

Complete search terms for Medline are included in Appendix A1, published as supplementary material online attached to the electronic version of this paper.

\section{Selection criteria}

Studies were included if they presented data on any CMD in a community or outpatient older population sample (aged $>50$ ), from South Asia. The age of 50 was used as a cutoff rather than the current Indian standard of 60 or the HIC standard of 65 as some well-known seminal studies conducted in the 1990s used this $50+$ cutoff and were considered relevant when planning this review. The South Asian countries included were Afghanistan, Bangladesh, Bhutan, Maldives, Nepal, India, Pakistan, and Sri Lanka. Only studies which used a tool, 


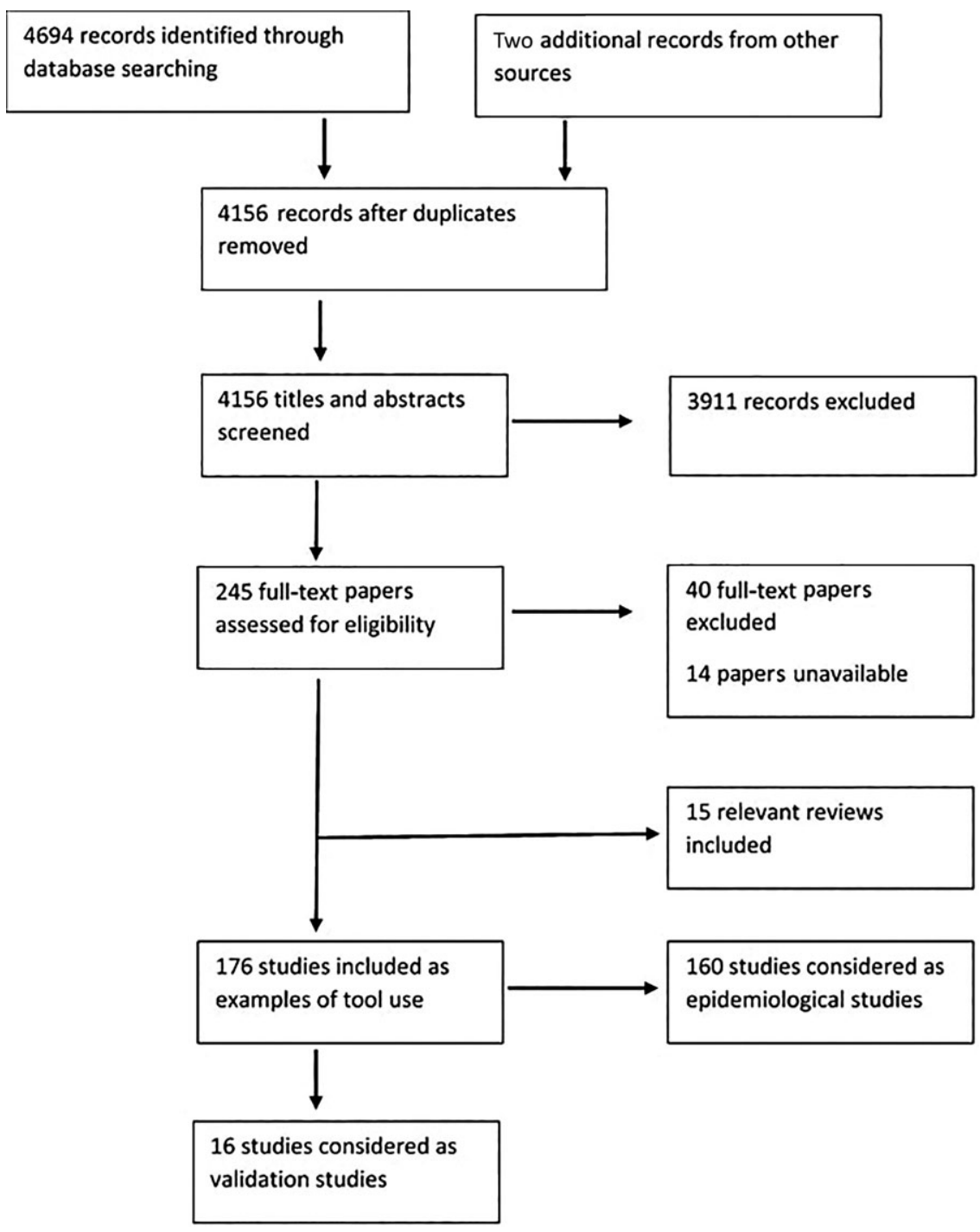

Figure 1. PRISMA flowchart.

questionnaire, or instrument to identify or screen for a clinically defined CMD were included. Studies that used self-reported diagnoses considered only well-being rather than a psychiatric diagnosis of CMD, or did not specify the age range of the sample were excluded. Systematic and other reviews and opinion pieces were examined for references and the scope and major findings of reviews included for discussion and context of the current knowledge base, however, no data were extracted. There was no specifier for study design to restrict to validation studies as a pilot search indicated that this would exclude papers known to be of interest from the search results. This allowed the collection of all instances of tool use rather than just those in which the purpose was validation. The search was limited to peer-reviewed journal papers published between 1990 and August 2019 in the English language.

Search results were independently screened for eligibility by two authors ( $\mathrm{LF}$ and $\mathrm{CN}$ ) in a two-step process; title and abstract followed by full-text screening, as documented in the PRISMA diagram (Figure 1) (Moher et al., 2009). Any disagreements were resolved via discussion and the input of a third reviewer $(\mathrm{EBM})$. The reference lists of the 176 studies included after full-text screening were hand-searched to identify any other potentially relevant studies.

\section{Data extraction and synthesis}

VAlidation studies

Studies presenting a new tool, or validating an existing tool were of primary interest and are presented in Table 1. Data extracted included: the country and region the study sample was drawn from; sample size (limited to participants in South Asia where several countries were included); setting (community dwelling, outpatient, or care home); urban or rural; $\mathrm{CMD}$ (s) assessed; name of tool, 
whether it was novel or previously developed and/or validated elsewhere and if so, where it was developed originally; language of the validated version.

Where the tool was compared to a reference standard, further information was extracted according to its performance as a diagnostic test (sensitivity, specificity, and cutoff value used) and gold standard assessment used for validation if relevant.

These studies were quality assessed using the QUADAS-2 (Whiting et al., 2011) by two authors (LF and EBM). In addition to the recommended signaling questions, one was added asking whether the approach to cultural adaptation or translation increased the risk of bias.

Epidemiological data

Data were also extracted from all epidemiological studies that used an instrument to diagnose a CMD, whether or not there was any validation described - see Table S1 published as supplementary material online attached to the electronic version of this paper. Data extracted included: demographic data on the population, participants, and study setting as above; study design; primary outcome of the study; $\mathrm{CMD}(\mathrm{s})$ assessed; tool used to identify CMD(s); type of tool (screening/diagnostic); where the tool was developed (HIC or LMIC); if the tool was validated for that context, and if not, where it was validated; any other significant features. These studies were not quality assessed other than to judge the cultural appropriateness of the tool used and no quantitative data were extracted . Their inclusion was intended to add to an understanding of how CMD screening tools are used in this context.

A proportion of the records, selected by systematic sampling, were independently reviewed to ensure the reliability of data extraction (S-MP). Due to temporary restrictions on access to library resources, 14 papers were not available. These were all epidemiological studies.

\section{Results}

\section{Validation studies}

Identification of novel tools or contextually adapted and validated tools was a central purpose of this review. A total of 15 tools aiming to meet this need were identified from the literature. One was a novel instrument developed in South Asia, and 14 were adaptations of existing instruments developed originally in HICs. Ten of these compared the adapted tool with an established gold standard. All tools which underwent a comparison with a gold standard in this way were for depression. These 10 tools were quality assessed using the QUADAS-2 (Whiting et al., 2011).

Novel tools

Only one entirely novel culturally specific tool was identified (Madnawat and Kachhawa, 2007) - aiming to specifically address death anxiety in urban community residents of Jaipur district, India. This scale was constructed de novo by local experts and so should be optimally relevant to this population. The study was not designed to assess any measures of validity, however, and so it is difficult to comment objectively on its merits compared to other, less culturally specific tools.

Adaptations OF HIC TOOLS AS INDEX TESTS WITH A REFERENCE STANDARD

Ten studies made use of an existing screening tool developed in a HIC, translated, and/or adapted this to a particular cultural context and validated through comparison to a gold standard. In seven of these, the gold standard was an expert clinical interview, and in the others, this was a structured diagnostic tool (Geriatric Mental state [GMS], Clinical Interview Schedule - Revised [CIS-R] or Structured Clinical Interview for DSM-IV [SCID]). Eight out of these 10 studies were from India, 1 from Nepal, and 1 from Sri-Lanka. All screened for depression. They are detailed in Table 1. It can be seen here that the diagnostic accuracy of specific tools as a diagnostic test varies substantially across different settings. Studies of GDS-15 adaptions report sensitivities of $73-100 \%$, and specificities of $48-94 \%$, although a range of cutoffs was used. Area under the receiver-operator characteristic (AUROC) value was not commonly reported. An analysis of the specific adaptions, which mostly affected performance was beyond the scope of this study.

The QUADAS-2 tool, a quality assessment tool for diagnostic accuracy studies (Whiting et al., 2011) highlights quality concerns or unclear reporting in all but 2 out of the 10 studies assessed (Table 2). The most common source of bias identified was lack of blinding, that is, the index test was administered with knowledge of the results of the reference standard, or vice versa. The approach to patient selection was often incompletely reported, explaining the frequently unclear risk of bias in this category.

OTHER MEASURES OF VALIDITY

A further four studies aimed to explore various measures of validity, but not examine performance as a diagnostic test. These were not included in the quality assessment. Two of these studies examined 
Table 1. Adaptations of HIC older age depression screening tools as index tests with a reference standard

\begin{tabular}{|c|c|c|c|c|c|c|c|c|c|c|c|}
\hline AUTHOR & LOCATION & $N$ & SETTING & CMD & $\begin{array}{c}\text { TOOL } \\
\text { ADAPTED }\end{array}$ & $\begin{array}{l}\text { TOOL } \\
\text { FROM }\end{array}$ & $\begin{array}{c}\text { GOLD } \\
\text { STANDARD }\end{array}$ & LANGUAGE & $\begin{array}{l}\text { CUTOFF } \\
(\geq)\end{array}$ & SENSITIVITY & SPECIFICITY \\
\hline $\begin{array}{l}\text { Prakash et al. } \\
\quad(2009)\end{array}$ & Bikaner, India & 104 & $\begin{array}{l}\text { OPD - gen med, } \\
\text { urban }\end{array}$ & Depression & GDS-15 & HIC & $\begin{array}{c}\text { Clinical } \\
\text { interview (ICD } \\
\text { DCR) }\end{array}$ & Hindi & $5 / 15$ & $100 \%$ & $94 \%$ \\
\hline $\begin{array}{l}\text { Biswas et al. } \\
\quad \text { (2009) }\end{array}$ & Vellore, India & 204 & $\begin{array}{l}\text { Community, } \\
\text { urban }\end{array}$ & Depression & PHQ-2 & HIC & CIS-R & Tamil & $2 / 2$ & $94 \%$ & $48 \%$ \\
\hline $\begin{array}{l}\text { Guerra et al. } \\
\quad \text { (2015) }\end{array}$ & Chennai + Vellore, India & 2002 & $\begin{array}{l}\text { Community, } \\
\text { urban }+ \text { rural }\end{array}$ & Depression & EURO-D & HIC & GMS & $\begin{array}{c}\text { Tamil }+ \\
\text { Hindi }\end{array}$ & $5 / 12$ & $\begin{array}{l}\text { Urban: } 97 \% \\
\text { Rural: } 91 \%\end{array}$ & $\begin{array}{l}\text { Urban: } 74 \% \\
\text { Rural: } 70 \%\end{array}$ \\
\hline $\begin{array}{l}\text { Kumar et al. } \\
\text { (2016) }\end{array}$ & Thrissur, India & 275 & Community, rural & Depression & CES-D & HIC & $\begin{array}{c}\text { Clinical } \\
\text { interview (ICD- } \\
10)\end{array}$ & Malayalam & $4 / 10$ & $98 \%$ & $79 \%$ \\
\hline $\begin{array}{l}\text { Barua and } \\
\quad \operatorname{Kar}(2010)\end{array}$ & Udupi district, India & 627 & Community, rural & Depression & WHO-5 & $\mathrm{HIC}$ & $\begin{array}{c}\text { Clinical } \\
\text { interview (ICD- } \\
10)\end{array}$ & $\begin{array}{l}\text { Kannada } \\
+ \text { Hindi }\end{array}$ & $\begin{array}{l}\text { Not } \\
\text { stated }\end{array}$ & $97 \%$ & $86 \%$ \\
\hline $\begin{array}{l}\text { Khattri and } \\
\text { Nepal } \\
(2006)\end{array}$ & Kathmandu, Nepal & 100 & $\begin{array}{l}\text { OPD - gen med } \\
+ \text { psych, urban }\end{array}$ & Depression & GDS-30 & HIC & $\begin{array}{c}\text { Clinical } \\
\text { interview }\end{array}$ & Nepali & $\begin{array}{l}\text { Not } \\
\text { stated }\end{array}$ & $85 \%$ & $82 \%$ \\
\hline $\begin{array}{l}\text { Kulathunga } \\
\text { et al. (2010) }\end{array}$ & Colombo, Sri Lanka & 30 & $\begin{array}{l}\text { OPD - psych, } \\
\text { urban }\end{array}$ & Depression & GDS-15 & HIC & $\begin{array}{c}\text { Clinical } \\
\text { interview (ICD- } \\
10)\end{array}$ & Sinhalese & $8 / 15$ & 73.3 & 73.3 \\
\hline $\begin{array}{l}\text { Sarkar et al. } \\
\quad(2015)\end{array}$ & Puducherry, India & 242 & Community, rural & Depression & GDS-15 & HIC & $\begin{array}{c}\text { Clinical } \\
\text { interview (ICD- } \\
10)\end{array}$ & Tamil & $8 / 15$ & $80 \%$ & $48 \%$ \\
\hline $\begin{array}{l}\text { Banerjee et al. } \\
\text { (2008) }\end{array}$ & Chandigarh, India & 53 & $\begin{array}{l}\text { OPD - ophthal., } \\
\text { urban }\end{array}$ & Depression & GDS-15 & HIC & SCID & Not stated & $5 / 15$ & $97 \%$ & $54 \%$ \\
\hline $\begin{array}{l}\text { Prince et al. } \\
\quad(2004)\end{array}$ & $\begin{array}{c}\text { Chennai x2, Goa, Thrissur, } \\
\text { Hyderabad, All India }\end{array}$ & 414 & $\begin{array}{l}\text { Community, } \\
\text { urban + rural }\end{array}$ & Depression & $\begin{array}{l}\text { GMS + } \\
\text { organicity } \\
\text { items }\end{array}$ & HIC & $\begin{array}{c}\text { Clinical } \\
\text { interview + } \\
\text { MADRS }\end{array}$ & Not stated & $\mathrm{n} / \mathrm{a}$ & $\mathrm{n} / \mathrm{a}$ & $\begin{array}{c}83 \%, 100 \%, \\
91 \%, 100 \%, \\
74 \%\end{array}$ \\
\hline
\end{tabular}

Prince et al. (2004) could not report sensitivity for the GMS for methodological reasons.

Abbreviations: OPD, outpatient department; GDS, Geriatric Depression Scale; HIC, high-income country; ICD, International Classification of Diseases; DCR, diagnostic criteria for research; PHQ,

patient health questionnaire; CIS-R, Clinical Interview Schedule - Revised; EURO-D, EURO - Depression; CES-D, Centre for Epidemiological Studies - Depression; WHO, World Health

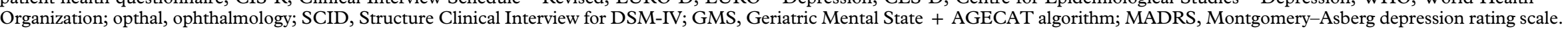


Table 2. QUADAS-2: quality assessment and risk of bias in diagnostic accuracy studies

\begin{tabular}{|c|c|c|c|c|c|c|c|}
\hline \multirow[b]{2}{*}{ STUDY } & \multicolumn{4}{|c|}{ RISK OF BIAS } & \multicolumn{3}{|c|}{ APPLICABILITY CONCERNS } \\
\hline & $\begin{array}{c}\text { PATIENT } \\
\text { SELECTION }\end{array}$ & $\begin{array}{l}\text { INDEX } \\
\text { TEST }\end{array}$ & $\begin{array}{l}\text { REFERENCE } \\
\text { STANDARD }\end{array}$ & $\begin{array}{l}\text { FLOW AND } \\
\text { TIMING }\end{array}$ & $\begin{array}{c}\text { PATIENT } \\
\text { SELECTION }\end{array}$ & $\begin{array}{l}\text { INDEX } \\
\text { TEST }\end{array}$ & $\begin{array}{l}\text { REFERENCE } \\
\text { STANDARD }\end{array}$ \\
\hline $\begin{array}{l}\text { Prakash et al. } \\
(2009)\end{array}$ & $\checkmark$ & $\checkmark$ & $\checkmark$ & $\checkmark$ & $\checkmark$ & $\checkmark$ & $\checkmark$ \\
\hline $\begin{array}{l}\text { Biswas et al. } \\
(2009)\end{array}$ & $\checkmark$ & $\checkmark$ & $?$ & $\checkmark$ & $\checkmark$ & $\checkmark$ & $\checkmark$ \\
\hline $\begin{array}{l}\text { Guerra et al. } \\
(2015)\end{array}$ & $\checkmark$ & $x$ & $x$ & $\checkmark$ & $\checkmark$ & $\checkmark$ & $\checkmark$ \\
\hline $\begin{array}{l}\text { Kumar et al. } \\
\text { (2016) }\end{array}$ & $?$ & $x$ & $x$ & $\checkmark$ & $\checkmark$ & $\checkmark$ & $\checkmark$ \\
\hline $\begin{array}{l}\text { Barua et al. } \\
\text { (2010) }\end{array}$ & $\checkmark$ & $x$ & $\checkmark$ & $x$ & $\checkmark$ & $\checkmark$ & $\checkmark$ \\
\hline $\begin{array}{l}\text { Khattri and Nepal } \\
\text { (2006) }\end{array}$ & $?$ & $x$ & $x$ & $\checkmark$ & $?$ & $\checkmark$ & $\checkmark$ \\
\hline $\begin{array}{l}\text { Kulathunga et al. } \\
\text { (2010) }\end{array}$ & $x$ & $\checkmark$ & $\checkmark$ & $\checkmark$ & $x$ & $\checkmark$ & $\checkmark$ \\
\hline $\begin{array}{l}\text { Sarkar et al. } \\
\quad(2015)\end{array}$ & $\checkmark$ & $\checkmark$ & $\checkmark$ & $\checkmark$ & $\checkmark$ & $\checkmark$ & $\checkmark$ \\
\hline $\begin{array}{l}\text { Prince et al. } \\
\quad(2004)\end{array}$ & $?$ & $\checkmark$ & $\checkmark$ & $\checkmark$ & $\checkmark$ & $\checkmark$ & $\checkmark$ \\
\hline $\begin{array}{l}\text { Banerjee et al. } \\
\text { (2008) }\end{array}$ & $\checkmark$ & $\checkmark$ & $\checkmark$ & $x$ & $\checkmark$ & $\checkmark$ & $\checkmark$ \\
\hline
\end{tabular}

$\checkmark$, Low Risk; $\boldsymbol{x}$, High Risk; ?, Unclear Risk.

scales, which were subsequently or previously compared to an appropriate gold standard in an equivalent setting (Chokkanathan and Mohanty, 2013; Ganguli et al., 1999) and another for the same culture but in a different context (clinic vs. community) (Gautam and Houde, 2011). Their related validations are included above. Thus, the most significant exclusion from quality assessment is the GAD-7 Urdu translation for an urban community in Pakistan (Ahmad et al., 2017).

\section{Screening tools utilized in epidemiological studies}

As part of a wider aim to scope research conduct in this area, we have reviewed the use of CMD diagnostic and screening tools used in existing relevant epidemiological research. This existing work covers a broad range of settings (Table 3), and a broadly representative geographical area (Figure 2). The majority of studies $(n=144,82 \%)$ measured only depression, and used the GDS $(n=95,54 \%)$ (Table 3), predominantly the 15 -item version. Only $57 \%$ used a tool that was culturally validated in line with WHO guidance (World Health Organisation, 2016), or with well-established cross-cultural applicability (e.g. GMS [Prince et al., 2004], CIDI [Haro et al., 2006]). Where a culturally validated screening tool was not used, most studies $(n=123$, $70 \%$ ) did not report a cultural adaptation or review of the tool used in the study methods, for example, using local translators and clinicians (see Table S1, published as supplementary material online attached to the electronic version of this paper).

\section{Reviews}

A total of 15 relevant reviews were identified in the search, including 7 meta-analyses, (Barua, 2013; Barua et al., 2010; Barua et al., 2011a; 2011b; Brandão et al., 2019; Grover and Malhotra, 2015; Parkar, 2015; Patel and Shaji, 2010; Pilania et al., 2019; Prakash et al., 2014; Qidwai and Ashfaq, 2011; Rao, 1993; Sami et al., 2015; Shaji et al., 2004; Thapa, 2019). Of the reviews identified, six were systematic reviews examining depression prevalence in India (Barua, 2013; Barua et al., 2010; Barua et al., 2011a; 2011b; Grover and Malhotra, 2015; Pilania et al., 2019). A further three systematic reviews assessed excess mortality in depression (Brandão et al., 2019), prevalence of all mental disorders in Nepal (Thapa, 2019), and the natural course of anxiety disorders (Sami et al., 2015). The remaining six reviews were narrative reviews describing elderly mental health in India (Prakash et al., 2014; Parkar, 2015; Shaji et al., 2004; 
Table 3. Summary characteristics of epidemiological data studies using screening and diagnostic tools

(a) STUDY LOCATION

\begin{tabular}{|c|c|c|c|c|}
\hline COUNTRY & NUMBER OF STUDIES & $\%$ OF TOTAL & POPULATION $\left(\times 10^{6}\right)$ & $\%$ OF SOUTH ASIAN POPULATION \\
\hline India & 132 & 75.0 & 1210 & 72.7 \\
\hline Pakistan & 23 & 13.1 & 212 & 12.7 \\
\hline Nepal & 10 & 5.7 & 26 & 1.6 \\
\hline Sri Lanka & 9 & 5.1 & 20 & 1.2 \\
\hline Bangladesh & 2 & 1.1 & 163 & 9.8 \\
\hline Afghanistan & 0 & 0 & 32 & 1.9 \\
\hline Bhutan & 0 & 0 & 0.7 & 0.04 \\
\hline Maldives & 0 & 0 & 0.4 & 0.02 \\
\hline
\end{tabular}

\begin{tabular}{|c|c|c|}
\hline STUDY SETTING & NUMBER OF STUDIES & $\%$ OF TOTAL \\
\hline Urban & 84 & 47.7 \\
\hline Rural & 43 & 24.4 \\
\hline Both separately & 21 & 11.9 \\
\hline Combined & 12 & 6.8 \\
\hline Not stated & 16 & 9 \\
\hline
\end{tabular}

(c) FREQUENCY OF MOST FREQUENTLY UTILIZED TOOLS

\begin{tabular}{|c|c|c|c|}
\hline NAME OF TOOL & $\begin{array}{c}\text { PURPOSE OF } \\
\text { TOOL }\end{array}$ & NUMBER OF STUDIES & $\%$ OF TOTAL \\
\hline GDS & Screening & 95 & 54.0 \\
\hline CES-D & Screening & 12 & 6.8 \\
\hline EURO-D & Screening & 7 & 4.0 \\
\hline PHQ & Screening & 6 & 3.4 \\
\hline GMS & Diagnostic & 15 & 8.5 \\
\hline CIDI & Diagnostic & 13 & 7.4 \\
\hline
\end{tabular}

Abbreviations: GDS, Geriatric Depression Scale; CES-D, Centre for Epidemiological Studies - Depression; EURO-D, EURO - Depression; PHQ, patient health questionnaire; GMS, Geriatric Mental State, CIDI, Composite International Diagnostic Interview.

Rao, 1993), Pakistan (Qidwai and Ashfaq, 2011), and South Asia as a whole (Patel and Shaji, 2010). There were no studies specifically on tool use. Data were not extracted systematically, however, findings relevant to this review are discussed below.

\section{Funding}

Seventy-three percent of the studies gave a statement regarding funding or conflict of interest. Funding was predominated by either local research grants or the funders of major epidemiological exercises such as the 10/66 Collaboration and the World Mental Health Survey.

\section{Unavailable papers}

Fourteen papers were not available in U.K. libraries and were unavailable for review. These were all small epidemiological studies, not anticipated to significantly impact findings.

\section{Discussion}

\section{Validation and adaptation of tools}

\section{REFERENCE STANDARDS}

In validating a screening tool, an expert diagnostic interview remains the preferred gold standard (Ali et al., 2016), but this is a scarce resource in many settings and a more practical compromise must often be found. The CIS-R, for example, is a structured, standardized interview, for use by lay interviewers to detect minor psychiatric disorders such as anxiety and depression. It has been shown to be highly reliable in other LMICs, for example, Chile (Lewis et al., 1992) which was then an LMIC, and 


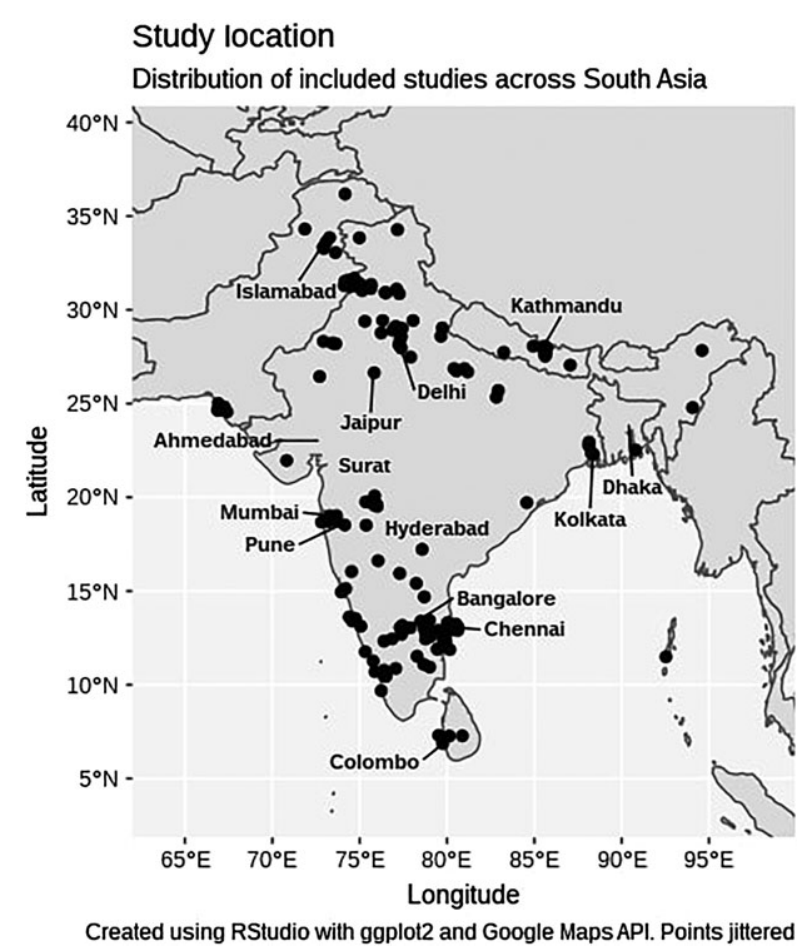

Figure 2. Map showing the distribution of all studies included in the review across South Asia.

Malaysia (Subramaniam et al., 2006), although the Indian context remains unassessed. Similarly, the GMS/AGECAT algorithm, as used by the 10/66 group (Prince et al., 2007) can produce a range of diagnoses. It is specifically designed for use by lay interviewers with older participants, accounts for cognitive impairment, and has performed well in LMICs including those where literacy rates may be low (Prince et al., 2004). More widespread validation of these promising tools would provide an important resource for researchers struggling with the lack of available expert interviewers.

\section{Adapting HIC tools For LMICs}

This review has identified a tendency to use unvalidated screening tools without an adaptation process in epidemiological studies, undermining their conclusions. The WHO (2016) provides a straightforward summary of how the process of translation and cultural adaptation might be done. This and similar adaptation processes are known to improve the performance of tools as screening instruments for CMD across LMICs (Ali et al., 2016). The GDS, in particular, required alteration to meet the needs of different cultures in a cross-cultural assessment of geriatric depression (Mui et al., 2001), though it has been identified as a screening tool well suited to the Indian context (Prakash et al., 2014) and performs well in the validation studies included in Table 1.
Advocating for resource allocation, and the design of effective public policy to meet the mental health needs of older persons in South Asia requires good quality data available locally (Arokiasamy et al., 2017; Ebrahim et al., 2013), especially in LMICs where mental health needs are often overlooked in favor of the many other pressing concerns (Brandão et al., 2019). In omitting any cultural adaptation, the resulting data will not take account of the important influence of culture, language, and education on the presentation of mental disorders (Kerr, 2001; Prince et al., 2007), and therefore the population's needs may be inadequately measured and provided for.

A meta-analysis of the performance of screening tools for CMD across LMICs found that tools specifically designed for a particular setting tend to perform better than equivalent tools adapted from a HIC, however, more limited data evaluating these restricted the conclusions they were able to draw in comparing them with HIC versions in wider use (Ali et al., 2016). This may be because cultural factors fundamentally affect how the disorder is experienced. Simple translations of HIC tools may miss out on the emic factors, which can provide an important dimension to understanding disorders as they manifest locally. No validated examples of this were identified in this review, however, examples from elsewhere include the Shona Symptom Questionnaire for Zimbabwe (Patel et al., 1997), the case description method for CMD in China (Sheng, 2010), the Chinese Military Mental Health Scale for CMD (Wang et al., 2012), and the Pakistan anxiety and depression questionnaire (Mumford et al., 2005). These are not designed specifically for the older population however.

\section{Reviews}

Limitations OF THE CURRENT RESEARCH BASE Reviews identified through searching highlight the shortage of high-quality research available in this area. The lack of appropriate diagnostic and screening tools available to researchers contributes to this shortcoming.

Meta-analyses have demonstrated a wide range of prevalence estimates for CMD in older people, particularly in India. This may be in excess of that found worldwide (Barua, 2013; Barua et al., 2011b; Pilania et al., 2019; World Health Organisation, 2001), however, these estimates are limited by methodological imprecision in the studies available for review (Barua et al., 2010; 2011b; Grover and Malhotra, 2015; Pilania et al., 2019). This limits the extent to which these reviews can usefully inform the provision of measures to reduce the mental health gap for older people in South Asia. 
A meta-analysis of the prevalence of depression in India (Pilania et al., 2019) found an overreliance on screening tools such as the Geriatric Depression Scale (GDS) and Centre for Epidemiological Studies - Depression Scale (CES-D) to make a diagnosis in epidemiological surveys, and noted an increased prevalence in studies relying on such instruments. Similarly, a meta-analysis in Nepal (Thapa, 2019) found the prevalence of mental disorders was significantly reduced when studies relying on screening tools were excluded, noting a particular reliance on the GDS.

There are therefore limited and imprecise data available to policymakers. In the data that do exist, there is an overreliance on screening tools used for diagnosis. Contributing to this imprecision is the lack of cultural validation in the screening tools that are used.

\section{OUTSIDE OF INDiA}

There was a particular lack of reviews concentrating on research outside of India. One meta-analysis found the prevalence of mental disorder in Nepal to be similar to that found in India if the analysis was limited to diagnostic interviews (Thapa, 2019). The one review focused on Pakistan covered older persons' mental health broadly and did not attempt a meta-analysis (Qidwai and Ashfaq, 2011).

\section{Lack of anxiety studies}

This review shows that there is a clear focus on depression over other disorders such as anxiety. This is evident in the validation studies and epidemiological surveys found. A review of older persons' mental health needs in India reported a common overlap of depression and anxiety with almost half of depressed older patients reporting significant anxiety symptoms (Parkar, 2015). Furthermore, a worldwide meta-analysis of longitudinal studies of anxiety disorders in older persons found a poorer prognosis of mixed anxiety and depression compared to pure anxiety or depression (Sami et al., 2015). The finding that $82 \%$ of the studies included in this review considered only depression and not anxiety therefore highlights a significant research gap. There was only one example of an anxietyspecific screening tool identified in this review (Ahmad et al., 2017), and this was not compared to a gold standard. This lack presents a significant challenge to researchers aiming to fill this gap.

\section{Limitations}

This review was intended to provide an overview of the literature on screening tools for CMD in older persons in South Asia, and with such a broad scope, the treatment of each area lacked the depth of analysis that would be required to make stronger conclusions. Some of these areas (e.g. prevalence data or the cultural adaptation of tools) would have benefited from a review or meta-analysis in their own right. Similarly, limiting the quality assessment only to validation studies assessed against a gold standard precludes comments on data quality in other areas. Only English language papers were included due to language restrictions of the authors. Grey literature was not reviewed.

\section{Conclusions and recommendations}

A number of recommendations follow from the research gaps identified in this review; that is in the lack of culturally validated diagnostic and screening tools for use throughout South Asia, and particularly for anxiety disorders which have been relatively neglected.

- The understanding of the mental health needs of older persons in South Asia still suffers from a scarcity of data, but also a lack of quality tools with which to tackle this. Validating appropriate tools should be a research priority.

- There is a severe lack of attention to anxiety disorders both as a contributor to disease burden, and in terms of tools available to researchers.

- The GDS appears to be the most suitable tool to adapt for screening older people for depression in this region, however, it has only been validated in a handful of settings, limiting its use.

- As well as screening tools, research in resource-constrained settings would benefit from local validations of diagnostic tools to substitute for expert clinical diagnosis where this is not available.

- There is a prominent lack of epidemiological studies in Bangladesh and of validation studies of any kind outside of India.

- There is likely to be value in developing novel tools for specific cultural contexts as these may outperform tools adapted from HICs.

\section{Conflicts of interest}

None.

\section{Source of funding}

Supported by a grant from the British Council (Grant no. 525227071). 


\section{Description of authors' roles}

LF - Database search, record screening (title and abstract), data extraction from full text, results analysis, critical appraisal, and primary author of the manuscript.

S-MP - Primary supervisor of the project, contributed to manuscript draft, review of data extraction, and project conception.

EBM - Methodology advice and supervision, final say where disagreement over inclusion, critical appraisal, and draft for methodology section of the manuscript.

$\mathrm{CN}$ - Record screening (title and abstract).

AL - Region-specific expertise, project conception, and input to manuscript drafts.

RW - Coordination of sharing of ideas between sites, project conception.

MV - Coordination of sharing of ideas between sites, project conception.

All authors approved the final version submitted.

\section{Acknowledgments}

We would like to acknowledge the North East England South Asia Mental health Alliance (NEESAMA), which made this collaboration possible and, in particular, to Dr Aditya Sharma and Professor Jacqui Rodgers for their support of the work of NEESAMA. We are especially grateful to Newcastle University, Cumbria, Northumberland, Tyne and Wear NHS Foundation Trust (CNTW) and the British Council for their financial support of NEESAMA.

\section{Supplementary material}

To view supplementary material for this article, please visit https://doi.org/10.1017/ S1041610220003804.

\section{References}

Agarwal, A., Lubet, A., Mitgang, E., Mohanty, S. and Bloom, D. (2016). Population Aging in India: Facts, Issues, and Options. PGDA Working Papers, 13216.

Ahmad, S., Hussain, S., Shah, F. S. and Akhtar, F. (2017). Urdu translation and validation of GAD-7: a screening and rating tool for anxiety symptoms in primary health care. IPMA. The fournal of the Pakistan Medical Association, 67, 1536-1540.

Ali, G.-c., Ryan, G. and Silva, M. J. D. (2016). Validated screening tools for common mental disorders in low and middle income countries: a systematic review. PloS One, 11, e0156939. doi: 10.1371/journal.pone.0156939
Arokiasamy, P. et al. (2017). Chronic noncommunicable diseases in 6 low- and middle-income countries: findings from wave 1 of the World Health Organization's Study on Global Ageing and Adult Health (SAGE). American fournal of Epidemiology, 185, 414-428. doi: 10.1093/aje/kww125

Banerjee, A., Kumar, S., Kulhara, P. and Gupta, A. (2008). Prevalence of depression and its effect on disability in patients with age-related macular degeneration. Indian fournal of Ophthalmology, 56, 469-474. doi: 10.4103/03014738.42643

Barua, A. (2013). Review article: trend of the prevalence of geriatric depression: a modern silent epidemic. Arquivos Brasileiros de Cardiologia, 100, 37-47.

Barua, A., Ghosh, M., Kar, N. and Basilio, M. (2010). Distribution of depressive disorders in the elderly. Fournal of Neurosciences in Rural Practice, 1, 67-73. doi: 10.4103/ 0976-3147.71719

Barua, A., Ghosh, M., Kar, N. and Basilio, M. (2011a). Prevalence of depressive disorder in the elderly. Annals of Saudi Medicine, 31, 620-624. doi: 10.4103/0256-4947 .87100

Barua, A., Ghosh, M. K., Kar, N. and Basilio, M. A. (2011b). Depressive disorders in elderly: an estimation of this public health problem. Fournal International Medical Sciences Academy, 24, 193-194.

Barua, A. and Kar, N. (2010). Screening for depression in elderly Indian population. Indian fournal of Psychiatry, 52, 150-153. doi: 10.4103/0019-5545.64595

Biswas, S. S. et al. (2009). Depression in the elderly in Vellore, South India: the use of a two-question screen. International Psychogeriatrics, 21, 369-371. doi: 10.1017/ S1041610208008259

Brandão, D. J., Fontenelle, L. F., da Silva, S. A., Menezes, P. R. and Pastor-Valero, M. (2019). Depression and excess mortality in the elderly living in low- and middle-income countries: systematic review and meta-analysis. International fournal of Geriatric Psychiatry, 34, 22-30. doi: 10.1002/gps.5008

Chand, M. (2018). Aging in South Asia: challenges and opportunities. South Asian fournal of Business Studies, 7, 189-206. doi: 10.1108/SAJBS-09-2017-0103

Chokkanathan, S. and Mohanty, J. (2013). Factor structure of the CES-D scale among older adults in Chennai, India. Aging $\mathcal{E}$ Mental Health, 17, 517-525. doi: 10.1080/13607863.2012.751580

Eaton, J. et al. (2011). Scale up of services for mental health in low-income and middle-income countries. The Lancet, 378, 1592-1603. doi: 10.1016/S0140-6736(11)60891-X

Ebrahim, S., Pearce, N., Smeeth, L., Casas, J. P., Jaffar, S. and Piot, P. (2013). Tackling non-communicable diseases in low- and middle-income countries: is the evidence from high-income countries all we need? PLoS Med, 10, e1001377. doi: 10.1371/journal.pmed.1001377

Ganguli, M., Dube, S., Johnston, J. M., Pandav, R., Chandra, V. and Dodge, H. H. (1999). Depressive symptoms, cognitive impairment and functional impairment in a rural elderly population in India: a Hindi version of the Geriatric Depression Scale (GDS-H). International fournal of Geriatric Psychiatry, 14, 807-820.

Gautam, R. and Houde, S. (2011). Geriatric Depression Scale for community-dwelling older adults in Nepal. Asian Fournal of Gerontology and Geriatrics, 6, 93-99. 
Grover, S. and Malhotra, N. (2015). Depression in elderly: review ofIndian research. FournalofGeriatricMentalHealth, 2, 4-15. doi: 10.4103/2348-9995.161376

Guerra, M., Ferri, C., Llibre, J., Prina, A. M. and Prince, M. (2015). Psychometric properties of EURO-D, a geriatric depression scale: a cross-cultural validation study. BMC Psychiatry, 15, 1-14. doi: 10.1186/s12888-0150390-4

Guerra, M. et al. (2016). A comparative cross-cultural study of the prevalence of late life depression in low and middle income countries. Fournal of Affective Disorders, 190, 362-368. doi: 10.1016/j.jad.2015.09.004

Haro, J. et al. (2006). Concordance of the Composite International Diagnostic Interview Version 3.0 (CIDI 3.0) with standardized clinical assessments in the WHO World Mental Health surveys. International fournal of Methods in Psychiatric Research, 15, 167-180. doi: 10.1002/mpr.196

Hay, S. I. et al. (2017). Global, regional, and national disability-adjusted life-years (DALYs) for 333 diseases and injuries and healthy life expectancy (HALE) for 195 countries and territories, 1990-2016: a systematic analysis for the Global Burden of Disease Study 2016. The Lancet, 390, 1260-1344. doi: 10.1016/S0140-6736(17)32130-X

Hossain, M. M., Purohit, N., Sultana, A., Ma, P., McKyer, E. L. J. and Ahmed, H. U. (2020). Prevalence of mental disorders in South Asia: an umbrella review of systematic reviews and meta-analyses. Asian fournal of Psychiatry, 51, 102041. doi: 10.1016/j.ajp.2020.102041

Kerr, L. (2001). Screening tools for depression in primary care. Culture and Medicine, 175, 349-352. doi: 10.1136/ ewjim.175.5.349

Khattri, J. B. and Nepal, M. K. (2006). Study of depression among geriatric population in Nepal. Nepal Medical College fournal: NMCF, 8, 220-223.

Kowal, P. et al. (2012). Data resource profile: the World Health Organization Study on global AGEing and adult health (SAGE). International fournal of Epidemiology, 41, 1639-1649. doi: 10.1093/ije/dys210

Kulathunga, M., Umayal, S., Somaratne, S., Srikanth, S., Kathriarachchi, S. and Krd, D. S. (2010). Validation of the Geriatric Depression Scale for an elderly Sri Lankan clinic population. Indian fournal of Psychiatry, 52, 254-256. doi: 10.4103/0019-5545.70979

Kumar, S., Nakulan, A., Thoppil, S. P., Parassery, R. P. and Kunnukattil, S. S. (2016). Screening for depression among community-dwelling elders: usefulness of the center for epidemiologic studies depression scale. Indian fournal of Psychological Medicine, 38, 483-485. doi: 10 .4103/0253-7176.191380

Lewis, G., Pelosi, A. J., Araya, R. and Dunn, G. (1992). Measuring psychiatric disorder in the community: a standardized assessment for use by lay interviewers. Psychological Medicine, 22, 465-486. doi: 10.1017/ S0033291700030415

Lotfaliany, M., Hoare, E., Jacka, F. N., Kowal, P. and Berk, M. (2019). Variation in the prevalence of depression and patterns of association, sociodemographic and lifestyle factors in community-dwelling older adults in six low- and middle-income countries. Fournal of Affective Disorders, 251, 218-226. doi: 10.1016/j.jad.2019.01.054

Madnawat, A. V. S. and Kachhawa, P. S. (2007). Age, gender, and living circumstances: discriminating older adults on death anxiety. Death Studies, 31, 763-769. doi: 10.1080/07481180701490743

Moher, D., Liberati, A., Tetzlaff, J., Altman, D. and Group, P. (2009). Preferred reporting items for systematic reviews and meta-analyses: the PRISMA statement. PLoS Med, 6, e1000097. doi: 10.1371/journal .pmed.1000097

Mui, A. C., Burnette, D. and Chen, L. M. (2001). Crosscultural assessment of geriatric depression: a review of the CES-D and the GDS. Fournal of Mental Health and Aging, 7, 137-164.

Mumford, D., Ayub, M., Karim, R., Izhar, N., Asif, A. and Bavington, J. (2005). Development and validation of a questionnaire for anxiety and depression in Pakistan. fournal of Affective Disorders, 88, 175-182. doi: 10.1016/j.jad .2005 .05 .015

Ogbo, F., Mathsyaraja, S., Koti, R., Perz, J. and Page, A. (2018). The burden of depressive disorders in South Asia, 1990-2016: findings from the global burden of disease study. BMC Psychiatry, 18, 333. doi: 10.1186/ s12888-018-1918-1

Parkar, S. R. (2015). Elderly mental health: needs. Mens Sana Monographs, 13, 91-99. doi: 10.4103/0973-1229 .153311

Patel, V. (2010). Mental illness in the elderly in South Asia. In M. T. Abou-Saleh, C. Katona and A. Kumar, (Eds.). Principles and Practice of Geriatric Psychiatry: Third Edition (pp. 730-733). Chichester: John Wiley and Sons. doi: 10 .1002/9780470669600.ch115

Patel, V., Simunyu, E., Gwanzura, F., Lewis, G. and Mann, A. (1997). The Shona Symptom Questionnaire: the development of an indigenous measure of common mental disorders in Harare. Acta Psychiatrica Scandinavica, 95, 469-475. doi: 10.1111/j.1600-0447.1997.tb10134.x

Pilania, M. et al. (2019). Prevalence of depression among the elderly (60 years and above) population in India, 19972016: a systematic review and meta-analysis. BMC Public Health, 19, 18. doi: 10.1186/s12889-019-7136-Z

Prakash, O., Gupta, L. N., Singh, V. B. and Nagarajarao, G. (2009). Applicability of 15-item Geriatric Depression Scale to detect depression in elderly medical outpatients. Asian fournal of Psychiatry, 2, 63-65. doi: 10 $.1016 /$ j.ajp.2009.04.005

Prakash, O., Shaji, K., Bharath, S. and Kumar, R. (2014). Research updates on late-life depression in India. Indian Fournal of Psychiatry, 56, S11.

Prince, M. et al. (2004). Effects of education and culture on the validity of the Geriatric Mental State and its AGECAT algorithm. The British fournal of Psychiatry, 185, 429-436. doi: 10.1192/bjp.185.5.429

Prince, M., Ferri, C. P., Acosta, D., Albanese, E., Arizaga, R. and Dewey, M. (2007). The protocols for the 10/66 dementia research group population-based research programme. BMC Public Health, 7, 165. doi: 10.1186/14712458-7-165

Qidwai, W. and Ashfaq, T. (2011). Elderly patients and their health in Pakistan: current status, issues, challenges and opportunities. Fournal of the Liaquat University of Medical and Health Sciences, 10.

Rama, M., Béteille, T., Li, Y., Mitra, P. and Newman, J. (2014). Addressing Inequality in South Asia. Washington, DC: The World Bank. 
Ranjan, J. and Asthana, H. (2017). Prevalence of mental disorders in India and other South Asian countries. Asian fournal of Epidemiology, 10, 45-53. doi: 10.3923/aje.2017.45.53

Rao, A. V. (1993). Psychiatry of old age in India. International Review of Psychiatry, 5, 165-170. doi: 10.3109/ 09540269309028307

Sami, M. B., Nilforooshan, R., Pachana, N. A. and Oude Voshaar, R. C. (2015). The natural course of anxiety disorders in the elderly: a systematic review of longitudinal trials. International Psychogeriatrics, 27, 1061-1069. doi: 10 $.1017 /$ S1041610214001847

Sarkar, S., Kattimani, S., Roy, G., Premarajan, K. C. and Sarkar, S. (2015). Validation of the Tamil version of short form Geriatric Depression Scale-15. Fournal of Neurosciences in Rural Practice, 6, 442-446. doi: 10.4103/ 0976-3147.158800

Shaji, K. S., Kishore, N. R. A., Lal, K. P., Pinto, C. and Trivedi, J. K. (2004). Better mental health care for older people in India. Indian fournal of Psychiatry, 46, 367-372.

Sheng, L. (2010). Better detection of non-psychotic mental disorders by case description method in China. Asian Fournal of Psychiaty, 3, 227-232. doi: 10.1016/j.ajp.2010 .07 .011

Subramaniam, K., Krishnaswamy, S., Jemain, A. A., Hamid, A. and Patel, V. (2006). The Clinical Interview Schedule-Revised (CIS-R)-Malay version, clinical validation. The Malaysian fournal of Medical Sciences, 13, 58-62.

Thapa, D. (2019). Prevalence of mental disorders among older people in Nepal: a systematic review. Kathmandu University Medical fournal, 16, 181-190.

Wang, Y., Zhang, L., Chen, C. and Yao, G. (2012). Application of Chinese Military Mental Health Scale in screening mental health of new recruits. Fournal of Chinese People's Liberation Army, 37, 420-424.

Whiting, P. et al. (2011). A revised tool for the quality assessment of diagnostic accuracy studies. Annals of Internal Medicine, 155, 529-536. doi: 10.7326/0003-4819-155-8201110180-00009

World Health Organisation (2001). Burden of mental and behavioural disorders. In A. Haden and B. Campanini (Eds.). The World Health Report 2001 - Mental Health: New Understanding, New Hope (pp. 19-44). Geneva, Swizerland: WHO.

World Health Organisation (2016). Management of substance abuse Research Tools: Process of translation and adaptation of instruments [Online]. Available at: https:// www.who.int/substance_abuse/research_tools/translation/ en/; last accessed 22 November 2020.

Yesavage, J. et al. (1982). Development and validation of a geriatric depression screening scale: a preliminary report. Fournal of Psychiatric Research, 17, 37-49. doi: 10.1016/ 0022-3956(82)90033-4 